PROCEEDINGS OF THE

AMERICAN MATHEMATICAL SOCIETY

Volume 139, Number 6, June 2011, Pages 2227-2237

S 0002-9939(2011)10811-6

Article electronically published on January 28, 2011

\title{
INTEGRAL REPRESENTATION OF SKOROKHOD REFLECTION
}

\author{
VENKAT ANANTHARAM AND TAKIS KONSTANTOPOULOS \\ (Communicated by Edward C. Waymire)
}

\begin{abstract}
We show that a certain integral representation of the one-sided Skorokhod reflection of a continuous bounded variation function characterizes the reflection in that it possesses a unique maximal solution which solves the Skorokhod reflection problem.
\end{abstract}

\section{INTRODUCTION}

The Skorokhod reflection problem has a long history. Skorokhod 10 introduced it as a method for representing a diffusion process with a reflecting boundary at zero. Given a continuous function $X:[0, \infty) \rightarrow \mathbb{R}$, the standard Skorokhod reflection problem seeks to find $(Q(t), t \geq 0)$ and a continuous, nondecreasing function $Y$ : $[0, \infty) \rightarrow \mathbb{R}_{+}$with $Y(0)=0$, such that $Q(t):=X(t)+Y(t) \geq 0$ for all $t$, and $\int_{0}^{\infty} Q(s) d Y(s)=0$. Intuitively, the latter expresses the idea that $Y$ can increase only at points $t$ such that $X(t)+Y(t)=0$. Skorokhod [10] showed that there is only one such $Y$, namely, $Y(t)=-\inf _{0 \leq s \leq t}(X(s) \wedge 0)$, and thus

$$
Q(t)=X(t) \vee \sup _{0 \leq s \leq t}(X(t)-X(s)) .
$$

We use the standard notation $a \vee b:=\max (a, b), a \wedge b:=\min (a, b)$. The mapping $X \mapsto Q$ is referred to as the (one-sided) Skorokhod reflection mapping and has now become a standard tool in probability theory and other areas. As an example, we recall that if $X$ is the path of a Brownian motion, then $Q$ is a reflecting Brownian motion and $Q(t)$ has the same distribution as $|X(t)|$ for all $t \geq 0$ 3, 9. Several extensions of the Skorokhod reflection mapping exist generalizing the range of $X$ (see, e.g., [1]) or its domain (see, e.g., [1]).

The question resolved in this paper was motivated by an application of the Skorokhod reflection in stochastic fluid queues [7, 6. Suppose that $A, C$ are two jointly stationary and ergodic random measures defined on a common probability space $(\Omega, \mathscr{F}, \mathbb{P})$, with intensities $a, c$, respectively, such that $a<c$. Then there exists a unique stationary and ergodic stochastic process $(Q(t), t \in \mathbb{R})$ defined on

Received by the editors May 21, 2010.

2010 Mathematics Subject Classification. Primary 60G17; Secondary 45G99, 90B05.

Key words and phrases. Skorokhod reflection, sample path properties, integral representation, storage systems.

The research of the first author was supported by the ARO MURI grant W911NF-08-1-0233, Tools for the Analysis and Design of Complex Multi-Scale Networks, by the NSF grants CCF0635372 and CNS-0910702, by Marvell Semiconductor Inc., and by the U. C. Discovery program.

The second author was supported in part by an EPSRC grant and by the Isaac Newton Institute for Mathematical Sciences. 
$(\Omega, \mathscr{F}, \mathbb{P})$ such that, for all $t_{0} \in \mathbb{R},\left(Q\left(t_{0}+t\right), t \geq 0\right)$ is the Skorokhod reflection of $\left(Q\left(t_{0}\right)+A\left(t_{0}, t_{0}+t\right]-C\left(t_{0}, t_{0}+t\right], t \geq 0\right)$. In addition, if the random measures $A, C$ have no atoms, then

$$
Q(t)=\int_{-\infty}^{t} \mathbf{I}(Q(s)>C(s, t]) d A(s),
$$

for all $t \in \mathbb{R}, \mathbb{P}$-almost surely. The latter equation was called an "integral representation" of Skorokhod reflection and extensions of it were formulated and proved in 6]. The integral representation was found to be useful in several applications, e.g. (i) in deriving the so-called Little's law for stochastic fluid queues [2], stating that $\mathbb{E}[Q(0)]=(a / c) \mathbb{E}_{A}[Q(0)]$, where $\mathbb{E}_{A}$ is expectation with respect to the Palm measure [4] of $\mathbb{P}$ with respect to $A$, and (ii) in deriving the form of the stationary distribution of a stochastic process derived from the local time of a Lévy process [5.

In an open problems session of the workshop on "New Topics at the Interface Between Probability and Communications" [8], the second author asked whether and in what sense (1.1) characterizes Skorokhod reflection. The question will be made precise in Section 2 below, where the main theorem, Theorem 2.1 which answers the question, is stated. In Section 3 the integral representation is explicitly proved, along with some auxiliary results, which are proved in order to make the paper self-contained. Finally, in Section 4 a proof of Theorem 2.1 is given. This requires a number of lemmas, all proved in the same section.

\section{THE PROBLEM}

Consider a locally finite signed measure $X$ on the Borel sets of $\mathbb{R}$. Assume that $X$ has no atoms, i.e. that $X(\{t\})=0$ for all $t \in \mathbb{R}$. Define

$$
Q^{*}(t):=\sup _{0 \leq s \leq t} X(s, t], \quad t \geq 0,
$$

where $X(s, t]=X((s, t])$ is the value of $X$ at the interval $(s, t] 1$ In particular,

$$
Q^{*}(0)=0 .
$$

Let $X(t):=X(0, t]$ and write (2.1) as

$$
Q^{*}(t)=X(t)-\inf _{0 \leq s \leq t} X(s) .
$$

The standard terminology [3, 12] is that $Q^{*}$ solves the Skorokhod reflection problem for the function $t \mapsto X(t)$.

Decompose $X$ as the difference of two locally finite nonnegative measures $A, C$, without atoms; i.e. write

$$
X=A-C .
$$

We stress that $A, C$ are not necessarily the positive and negative parts of $X$. In other words, the decomposition is not unique. For instance, we can add an arbitrary locally finite nonnegative measure without atoms to both $A$ and $C$.

\footnotetext{
${ }^{1}$ Since $X, A, C$ are assumed to have no atoms, we may as well write $X[s, t]$ or $X(s, t)$ instead of $X(s, t]$, and likewise for $A$ and $C$, but we have chosen the notation to be consistent with possible generalizations.
} 
In [6] it was proved that (2.1) also satisfies the fixed point equation referred to as the "integral representation" of the reflected process:

$$
Q(t)=\int_{0}^{t} \mathbf{1}(Q(s)>C(s, t]) d A(s), \quad t \geq 0 .
$$

A simpler version of this appeared earlier in [7]; this version was concerned with the case where $C$ is a multiple of the Lebesgue measure. In an open problems session of the workshop on "New Topics at the Interface between Probability and Communications" [8], the second author asked whether and in what sense (2.3) implies (2.1); the question was actually asked for the special case where $C$ is a multiple of the Lebesgue measure.

In this note we answer this question by proving the following:

Theorem 2.1. Let $A, C$ be locally finite Borel measures on $\mathbb{R}_{+}=[0, \infty)$ without atoms and consider the integral equation (2.3). This integral equation admits a unique maximal solution, i.e. a solution which pointwise dominates any other solution. Further, this maximal solution is precisely the function $Q^{*}$ defined by (2.1).

We proceed as follows. First, we present some auxiliary results and also give a proof of (2.1) $\Rightarrow(2.3)$ which is different from the one found in [6. Then we prove Theorem 2.1 by a successive approximation scheme and by proving a number of lemmas.

\section{Proof of the integral Representation AND AUXILIARY RESULTS}

We first exhibit some properties of $Q^{*}$, defined by (2.1), and also show that $Q^{*}$ satisfies the integral equation (2.3). The proof of the latter in the special case where $C$ is a multiple of the Lebesgue measure can be found in [7, Lemma 1] and in [2, §3.5.3]. A more general case is dealt with in [6, Theorem 1]. We give a different proof in Proposition 3.4 below. The lemmas below are straightforward and wellknown, but we give proofs for completeness. As before, $X$ is a locally finite Borel measure without atoms and $X=A-C$ is a decomposition as the difference of two nonnegative locally finite Borel measures without atoms. We set

$$
A(t):=A(0, t], \quad C(t):=C(0, t] .
$$

Lemma 3.1. If $0 \leq s \leq s^{\prime} \leq t$ and if $Q^{*}(s)>C(s, t]$, then $Q^{*}\left(s^{\prime}\right)>C\left(s^{\prime}, t\right]$.

Proof. Assume that $C(s, t]<Q^{*}(s)=\sup _{0 \leq u \leq s} X(u, s]$. This is equivalent to

$$
\begin{aligned}
& C(t)-C(s)<\sup _{0 \leq u \leq s}\{A(s)-A(u)-(C(s)-C(u))\} \\
& =A(s)+\sup _{0 \leq u \leq s}\{-A(u)+C(u)\}-C(s),
\end{aligned}
$$

The right-hand side of the latter is increasing in $s$ and so replacing $s$ by a larger $s^{\prime}$ we obtain

$$
C(t)<A\left(s^{\prime}\right)+\sup _{0 \leq u \leq s^{\prime}}\{-A(u)+C(u)\}
$$

which is equivalent to $Q^{*}\left(s^{\prime}\right)>C\left(s^{\prime}, t\right]$. 
Lemma 3.2. $Q^{*}$ satisfies

$$
Q^{*}(t)=\sup _{s \leq u \leq t} X(u, t] \vee\left(Q^{*}(s)+X(s, t]\right), \quad 0 \leq s \leq t .
$$

Proof. We show that the right-hand side of (3.1) equals the left-hand side:

$$
\begin{aligned}
\sup _{s \leq u \leq t} X(u, t] \vee\left(Q^{*}(s)+X(s, t]\right) & =\sup _{s \leq u \leq t} X(u, t] \vee\left\{\left(\sup _{0 \leq u \leq s} X(u, s]\right)+X(s, t]\right\} \\
& =\sup _{s \leq u \leq t} X(u, t] \vee \sup _{0 \leq u \leq s}\{X(u, s]+X(s, t]\} \\
& =\sup _{s \leq u \leq t} X(u, t] \vee \sup _{0 \leq u \leq s} X(u, t] \\
& =\sup _{0 \leq u \leq t} X(u, t]=Q^{*}(t)
\end{aligned}
$$

Lemma 3.3. If $0 \leq s \leq t$ and if $Q^{*}(s) \geq C(s, t]$, then $Q^{*}(t)=Q^{*}(s)+X(s, t]$.

Proof. We use equation (3.1), rewritten as follows:

$$
Q^{*}(t)=\sup _{s \leq u \leq t}\left\{X(u, t] \vee\left(Q^{*}(s)+X(s, t]\right)\right\} .
$$

Suppose $0 \leq s \leq u \leq t$ and that $Q^{*}(s) \geq C(s, t]$. Then $Q^{*}(s) \geq C(s, u]$ and so

$$
\begin{aligned}
Q^{*}(s)+X(s, t] & \geq C(s, u]+X(s, t] \\
& =C(s, u]+A(s, t]-C(s, t] \\
& =A(s, t]-C(u, t] \\
& \geq A(u, t]-C(u, t]=X(u, t],
\end{aligned}
$$

and this inequality implies that the term $X(u, t]$ inside the bracket of the right-hand side of (3.2) is not needed. Hence $Q^{*}(t)=Q^{*}(s)+X(s, t]$, which is what we wanted to prove.

Define next

$$
\sigma^{*}(t):=\sup \left\{0 \leq s \leq t: Q^{*}(s) \leq C(s, t]\right\} .
$$

By Lemma 3.1 .

$$
\begin{array}{ll}
Q^{*}(s) \leq C(s, t], & \text { if } 0 \leq s \leq \sigma^{*}(t), \\
Q^{*}(s)>C(s, t], & \text { if } \sigma^{*}(t)<s \leq t,
\end{array}
$$

provided that the last inequality is nonvacuous. Since the function $Q^{*}$ is nonnegative and continuous, we also have

$$
Q^{*}\left(\sigma^{*}(t)\right)=C\left(\sigma^{*}(t), t\right] .
$$

Theorem 3.4. If $X$ is a locally finite signed Borel measure on $[0, \infty)$ without atoms and if $X=A-C$ is any decomposition of $X$ as the difference of two nonnegative locally finite Borel measures without atoms, then the function $Q^{*}$ defined by (2.1) satisfies (2.3). 
Proof. By Lemma 3.3, and the last display,

$$
\begin{aligned}
Q^{*}(t) & =Q^{*}\left(\sigma^{*}(t)\right)+A\left(\sigma^{*}(t), t\right]-C\left(\sigma^{*}(t), t\right] \\
& =A\left(\sigma^{*}(t), t\right] \\
& =\int_{\sigma^{*}(t)}^{t} d A(s) \\
& =\int_{0}^{t} \mathbf{1}\left(Q^{*}(s)>C(s, t]\right) d A(s),
\end{aligned}
$$

which is the integral representation formula (2.3). Note that, to obtain the last equality in the last display, we used (3.4a) $-(3.4 \mathrm{~b})$.

\section{Proof of Theorem 2.1}

A priori, it is not clear that (2.3) admits a maximal solution and, even if it does, whether it satisfies (2.1). We shall show the validity of these claims in the sequel.

We fix two locally finite measures $A$ and $C$ and define the map $\Theta$ on the set of nonnegative measurable functions by

$$
\Theta(Q)(t):=\int_{0}^{t} \mathbf{I}(Q(s)>C(s, t]) d A(s), \quad t \geq 0 .
$$

The integral equation (2.3) then reads

$$
Q=\Theta(Q) .
$$

We observe that $\Theta$ is increasing:

$$
\text { If } Q \leq \widetilde{Q} \text {, then } \Theta(Q) \leq \Theta(\widetilde{Q}) .
$$

Here, and in the sequel, given two functions $f, g:[0, \infty) \rightarrow \mathbb{R}$, we write $f \leq g$ to mean that $f(t) \leq g(t)$ for all $t \geq 0$. To see that (4.2) holds, simply observe that $Q \leq \widetilde{Q}$ implies $\mathbf{l}(Q(s)>C(s, t]) \leq \mathbf{l}(\widetilde{Q}(s)>C(s, t])$ for all $0 \leq s \leq t$.

Define next a sequence of functions $\left(Q_{k}, k=0,1,2, \ldots\right)$ by first letting

$$
Q_{0}:=\infty
$$

and then, recursively,

$$
Q_{k+1}:=\Theta\left(Q_{k}\right), \quad k \geq 0 .
$$

Clearly, $Q_{1}(t)=\int_{0}^{t} d A(s)=A(t)$. So $Q_{0} \geq Q_{1}$. Since $\Theta$ is an increasing map, we see that

$$
Q_{k} \geq Q_{k+1} \geq 0, \quad k \geq 0
$$

We can then define

$$
Q_{\infty}(t):=\lim _{k \rightarrow \infty} Q_{k}(t) .
$$

Lemma 4.1. If $Q=\Theta(Q)$, then $Q \leq Q_{\infty}$. Furthermore,

$$
Q^{*} \leq Q_{\infty} .
$$

Proof. Suppose that $Q$ satisfies $Q=\Theta(Q)$. Since the integrand in the right-hand side of (4.1) is $\leq 1$, we have $Q(t) \leq A(t)$ for all $t \geq 0$. Letting $\Theta^{(k)}$ be the $k$-fold composition of $\Theta$ with itself, we have

$$
Q=\Theta^{(k)}(Q) \leq \Theta^{(k)}(A)=Q_{k},
$$


and so $Q \leq Q_{\infty}$. In particular, Proposition 3.4 states that $Q^{*}=\Theta\left(Q^{*}\right)$. Hence $Q^{*} \leq Q_{\infty}$

However, it is not yet clear at this point that $Q_{\infty}$ is a fixed point of $\Theta$. We can only show that

$$
Q_{\infty} \geq \Theta\left(Q_{\infty}\right)
$$

Indeed, $Q_{\infty} \leq Q_{k}$ for all $k$, and so $\mathbf{l}\left(Q_{\infty}(s)>C(s, t]\right) \leq \mathbf{l}\left(Q_{k}(s)>C(s, t]\right)$, for all $0 \leq s \leq t$, implying that $\Theta\left(Q_{\infty}\right) \leq \Theta\left(Q_{k}\right)=Q_{k+1}$, and, by taking limits, that $\Theta\left(Q_{\infty}\right) \leq Q_{\infty}$

Definition 4.2 (Regulating functions). Consider functions $B:[0, \infty) \rightarrow[0, \infty)$ which are continuous, nondecreasing, with $B(0)=0$, such that $X(0, t]+B(t) \geq 0$ for all $t \geq 0$. Call these functions regulating functions of $X$. The set of regulating functions is denoted by $\mathcal{R}$.

We define a mapping

$$
\Phi: \mathcal{R} \rightarrow \mathcal{R}
$$

in two steps as follows.

Step 1. Given $B \in \mathcal{R}$, first define

$$
\sigma_{B}(t):=\sup \{0 \leq s \leq t: A(s)+B(s)-C(t) \leq 0\}, \quad t \geq 0 .
$$

To motivate this definition, note that if $B$ is chosen according to the formula $B(t)=$ $-\inf _{0 \leq s \leq t}\{A(s)-C(s)\}$, then $\sigma_{B}(t)=\sigma^{*}(t)$ for all $t$, where $\sigma^{*}$ was defined in (3.3).

Step 2. Then let

$$
\Phi(B)(t):=B\left(\sigma_{B}(t)\right), \quad t \geq 0 .
$$

We actually need to show that what is claimed in (4.3) holds, namely:

Lemma 4.3. If $B \in \mathcal{R}$, then $\Phi(B) \in \mathcal{R}$.

Proof. Clearly, $\sigma_{B}(\cdot)$ is nondecreasing. Since $B$ is nondecreasing, it follows that $\Phi(B)=B \circ \sigma_{B}$ is nondecreasing. Also, $\Phi(B)(0)=B\left(\sigma_{B}(0)\right)=B(0)=0$. From the continuity of $A, B$ and the definition of $\sigma_{B}$, we have

$$
A\left(\sigma_{B}(t)\right)+B\left(\sigma_{B}(t)\right)=C(t), \quad t \geq 0 .
$$

We also have

$$
\begin{aligned}
A(t)+\Phi(B)(t)-C(t) & =A(t)+B\left(\sigma_{B}(t)\right)-C(t) \\
& =\left[A(t)-A\left(\sigma_{B}(t)\right)\right]+\left[A\left(\sigma_{B}(t)\right)+B\left(\sigma_{B}(t)\right)-C(t)\right] \\
& =A(t)-A\left(\sigma_{B}(t)\right) \geq 0,
\end{aligned}
$$

where we used (4.4) in the third step. It remains to show that $\Phi(B)(\cdot)$ is continuous. Note that $\sigma_{B}(\cdot)$ need not be continuous. However, $C(\cdot)$ is a continuous function and so, by (4.4), $t \mapsto A\left(\sigma_{B}(t)\right)+B\left(\sigma_{B}(t)\right)$ is continuous. Hence

$$
\left[A\left(\sigma_{B}(t+)\right)-A\left(\sigma_{B}(t-)\right]+\left[B\left(\sigma_{B}(t+)\right)-B\left(\sigma_{B}(t-)\right)\right]=0, \quad \text { for all } t .\right.
$$

Since $A\left(\sigma_{B}(\cdot)\right)$ and $B\left(\sigma_{B}(\cdot)\right)$ are both nondecreasing, it follows that $A\left(\sigma_{B}(t+)\right)-$ $A\left(\sigma_{B}(t-) \geq 0\right.$ and $B\left(\sigma_{B}(t+)\right)-B\left(\sigma_{B}(t-)\right) \geq 0$ and, since their sum is zero, they are both zero, implying that $A\left(\sigma_{B}(\cdot)\right)$ and $B\left(\sigma_{B}(\cdot)\right)$ are continuous. 
An immediate property of $\Phi$ is that

$$
\Phi(B) \leq B \quad \text { for all } B \in \mathcal{R} .
$$

Indeed, for all $t \geq 0, \sigma_{B}(t) \leq t$ and so $B\left(\sigma_{B}(t)\right) \leq B(t)$.

Starting with the function

$$
B_{1}(t):=C(t), \quad t \geq 0,
$$

we recursively define

$$
B_{k+1}:=\Phi\left(B_{k}\right), \quad k \geq 1
$$

Therefore

$$
B_{1} \geq B_{2} \geq \cdots \geq B_{k} \downarrow B_{\infty}, \quad \text { as } k \rightarrow \infty,
$$

where the inequalities and the limit are pointwise.

Lemma 4.4. The function $B_{\infty}$, defined via (4.6), (4.7) and (4.8), is a member of the class $\mathcal{R}$.

Proof. $B_{\infty}$ is nondecreasing since all the $B_{k}$ are nondecreasing. Also, $B_{\infty}(0)=0$. Since for all $k, A+B_{k}-C \geq 0$, we have $A+B_{\infty}-C \geq 0$. We proceed to show that $B_{\infty}$ is a continuous function. We observe that, for $0 \leq t \leq t^{\prime}$,

$$
\begin{aligned}
\left|\Phi(B)\left(t^{\prime}\right)-\Phi(B)(t)\right| & =\left|B\left(\sigma_{B}\left(t^{\prime}\right)\right)-B\left(\sigma_{B}(t)\right)\right| \\
& =B\left(\sigma_{B}\left(t^{\prime}\right)\right)-B\left(\sigma_{B}(t)\right) \\
& \leq A\left(\sigma_{B}\left(t^{\prime}\right)\right)-A\left(\sigma_{B}(t)\right)+B\left(\sigma_{B}\left(t^{\prime}\right)\right)-B\left(\sigma_{B}(t)\right) \\
& =\left[A\left(\sigma_{B}\left(t^{\prime}\right)\right)+B\left(\sigma_{B}\left(t^{\prime}\right)\right)\right]-\left[A\left(\sigma_{B}(t)\right)+B\left(\sigma_{B}(t)\right)\right] \\
& =C\left(t^{\prime}\right)-C(t),
\end{aligned}
$$

where we again used (4.4). It follows that the family of functions $\{\Phi(B), B \in \mathcal{R}\}$ is uniformly bounded and equicontinuous on each compact interval of the real line. By the Arzelà-Ascoli theorem, the family is compact and therefore $B_{\infty}$ is continuous. We have established that $B_{\infty} \in \mathcal{R}$.

We now claim that $B_{\infty}$ is a fixed point of $\Phi$.

Lemma 4.5. $\Phi\left(B_{\infty}\right)=B_{\infty}$.

Proof. By definition,

$$
\Phi\left(B_{\infty}\right)(t)=B_{\infty}\left(\sigma_{B_{\infty}}(t)\right)
$$

where

$$
\sigma_{B_{\infty}}(t)=\sup \left\{0 \leq s \leq t: A(s)+B_{\infty}(s) \leq C(t)\right\} .
$$

Now, since $B_{k} \geq B_{k+1}$ for all $k \geq 1$, it follows that $\sigma_{B_{k}} \leq \sigma_{B_{k+1}}$ for all $k \geq 1$, and so

$$
\sigma_{L}(t):=\lim _{k \rightarrow \infty} \sigma_{B_{k}}(t)
$$

is well-defined. Since $B_{k} \geq B_{\infty}$ for all $k \geq 1$, we have $\sigma_{B_{k}} \leq \sigma_{B_{\infty}}$. Taking limits, we find

$$
\sigma_{L} \leq \sigma_{B_{\infty}}
$$


Using the last two displays and the fact that $B_{k}$ and $B_{\infty}$ are nondecreasing, we have

$$
\begin{aligned}
\Phi\left(B_{\infty}\right)(t)=B_{\infty}\left(\sigma_{B_{\infty}}(t)\right) & \geq B_{\infty}\left(\sigma_{L}(t)\right) \\
& =\lim _{k \rightarrow \infty} B_{k}\left(\sigma_{L}(t)\right) \\
& \geq \lim _{k \rightarrow \infty} B_{k}\left(\sigma_{B_{k}}(t)\right) \\
& =\lim _{k \rightarrow \infty} B_{k+1}(t)=B_{\infty}(t) .
\end{aligned}
$$

By inequality (4.5), $\Phi(B) \leq B$ for all $B \in \mathcal{R}$ and since, by Lemma 4.4, $B_{\infty} \in \mathcal{R}$, it follows that we also have $B_{\infty} \leq \Phi\left(B_{\infty}\right)$. Therefore $B_{\infty}=\Phi\left(B_{\infty}\right)$, as claimed.

Lemma 4.6. Consider the function $Q^{*}$ defined by (2.1) and define a function $B^{*}$ by

$$
B^{*}(t):=Q^{*}(t)-X(0, t], \quad t \geq 0
$$

Then

(i) $B^{*} \in \mathcal{R}$;

(ii) $B^{*}=\Phi\left(B^{*}\right)$.

Proof. (i) We have $X(0, t]+B^{*}(t)=Q^{*}(t) \geq 0$ for all $t$. Using (2.1) and (2.2) we see that

$$
B^{*}(t)=\sup _{0 \leq s \leq t}\{-A(s)+C(s)\} .
$$

Therefore, $B^{*}(0)=0$, and $B^{*}$ is continuous and nondecreasing. We conclude that $B^{*} \in \mathcal{R}$. To prove (ii), recall that $\Phi\left(B^{*}\right)=B^{*} \circ \sigma_{B^{*}}$, where

$$
\sigma_{B^{*}}(t)=\sup \left\{0 \leq s \leq t: A(s)+B^{*}(s) \leq C(t)\right\} .
$$

Splitting the supremum in (4.9) into two parts, we obtain

$$
\begin{aligned}
B^{*}(t) & =\sup _{0 \leq s \leq \sigma_{B^{*}}(t)}\{-A(s)+C(s)\} \vee \sup _{\sigma_{B^{*}}(t) \leq s \leq t}\{-A(s)+C(s)\} . \\
& =B^{*}\left(\sigma_{B^{*}}(t)\right) \vee \sup _{\sigma_{B^{*}}(t) \leq s \leq t}\{-A(s)+C(s)\} .
\end{aligned}
$$

For $s \geq \sigma_{B^{*}}(t)$, we have $A(s)+B^{*}(s) \geq C(t)$, i.e. $-A(s)+C(s) \leq B^{*}(s)-C(s, t]$. Therefore

$$
\begin{aligned}
B^{*}(t) & \leq B^{*}\left(\sigma_{B^{*}}(t)\right) \vee \sup _{\sigma_{B^{*}}(t) \leq s \leq t}\left\{B^{*}(s)-C(s, t]\right\} \\
& =B^{*}\left(\sigma_{B^{*}}\right)(t)=\Phi\left(B^{*}\right)(t) .
\end{aligned}
$$

Thus, $B^{*} \leq \Phi\left(B^{*}\right)$. On the other hand, since $B^{*} \in \mathcal{R}$, we have $\Phi\left(B^{*}\right) \leq B^{*}$, by (4.5).

Lemma 4.7. Let $B \in \mathcal{R}$ be any fixed point of $\Phi$. Then $B \leq B^{*}$.

Proof. Since $B=\Phi(B)=B \circ \sigma_{B}$ we have

$$
B=B \circ \sigma_{B}^{(k)},
$$

where $\sigma_{B}^{(k)}:=\underbrace{\sigma_{B^{\circ} \cdots \circ \sigma_{B}}}_{k \text { times }}$. Since

$$
t \geq \sigma_{B}(t) \geq \sigma_{B} \circ \sigma_{B}(t) \geq \cdots \geq \sigma_{B}^{(k)}(t)
$$


we may define

$$
\sigma_{B}^{(\infty)}(t):=\lim _{k \rightarrow \infty} \sigma_{B}^{(k)}(t)
$$

By the continuity of $B$,

$$
B=B \circ \sigma_{B}^{(\infty)}
$$

On the other hand, (4.4) gives

$$
A \circ \sigma_{B}^{(k+1)}+B \circ \sigma_{B}^{(k+1)}=C \circ \sigma_{B}^{(k)}, \quad k \geq 1 .
$$

Taking the limit as $k \rightarrow \infty$, and using the continuity of $A, B$ and $C$, we have

$$
A \circ \sigma_{B}^{(\infty)}+B \circ \sigma_{B}^{(\infty)}=C \circ \sigma_{B}^{(\infty)} .
$$

Since $A(t)+B^{*}(t) \geq C(t)$ for all $t$, we have

$$
A \circ \sigma_{B}^{(\infty)}+B^{*} \circ \sigma_{B}^{(\infty)} \geq C \circ \sigma_{B}^{(\infty)}
$$

and from the last two displays we conclude that

$$
B^{*} \circ \sigma_{B}^{(\infty)} \geq B \circ \sigma_{B}^{(\infty)} .
$$

Since $B^{*}$ is nondecreasing and since (4.10) holds, we have

$$
B^{*} \geq B^{*} \circ \sigma_{B}^{(\infty)} \geq B \circ \sigma_{B}^{(\infty)}=B,
$$

as claimed.

We are now ready to prove Theorem 2.1. We already know from Lemma 4.1 that $Q^{*} \leq Q_{\infty}$. So we only have to prove the opposite inequality. Recall that $Q_{1}=A$ and $B_{1}=C$. Trivially then

$$
Q_{1}(t)+C(t)=A(t)+B_{1}(t), \quad t \geq 0 .
$$

Thus, for $0 \leq s \leq t$ we have

$$
\begin{aligned}
Q_{1}(s)>C(s, t] & \Longleftrightarrow Q_{1}(s)+C(s)>C(t) \\
& \Longleftrightarrow A(s)+B_{1}(s)>C(t) \\
& \Longleftrightarrow s>\sigma_{B_{1}}(t) .
\end{aligned}
$$

From this we get

$$
\begin{aligned}
Q_{2}(t) & =\int_{0}^{t} \mathbf{1}\left(Q_{1}(s)>C(s, t]\right) d A(s) \\
& =\int_{0}^{t} \mathbf{1}\left(s>\sigma_{B_{1}}(t)\right) d A(s) \\
& =A(t)-A\left(\sigma_{B_{1}}(t)\right) .
\end{aligned}
$$

But (4.4) gives

$$
A\left(\sigma_{B_{1}}(t)\right)+B_{1}\left(\sigma_{B_{1}}(t)\right)=C(t),
$$

and so

$$
Q_{2}(t)+C(t)=A(t)+B_{1}\left(\sigma_{B_{1}}(t)\right)=A(t)+B_{2}(t), \quad t \geq 0 .
$$


We now claim that

$$
Q_{k}(t)+C(t)=A(t)+B_{k}(t), \quad t \geq 0, \quad k \geq 1 .
$$

This can be proved by induction along the same lines as above. Taking limits as $k \rightarrow \infty$, we conclude

$$
Q_{\infty}(t)+C(t)=A(t)+B_{\infty}(t), \quad t \geq 0 .
$$

Lemma 4.5 tells us that $B_{\infty}$ is a fixed point of $\Phi$, and so, by Lemma 4.7

$$
B_{\infty} \leq B^{*}
$$

Hence

$$
\begin{aligned}
Q_{\infty}(t)+C(t) & =A(t)+B_{\infty}(t) \\
& \leq A(t)+B^{*}(t) \\
& =Q^{*}(t)+C(t), \quad t \geq 0
\end{aligned}
$$

and this gives

$$
Q_{\infty} \leq Q^{*}
$$

as needed.

\section{ACKNOWLEDGMENT}

We thank the Isaac Newton Institute for Mathematical Sciences for providing the stimulating research atmosphere where this research work was done.

\section{REFERENCES}

1. V. Anantharam and T. Konstantopoulos, Regulating functions on partially ordered sets, Order 22 (2005), 145-183. MR2207195 (2007g:06001)

2. F. Baccelli and P. Brémaud, Elements of Queueing Theory, Springer-Verlag, New York, 2003. MR $1957884(2003 \mathrm{~m}: 60001)$

3. R. Williams and K.-L. Chung, An Introduction to Stochastic Integration, Birkhäuser, Boston, 1989. MR 1102676 (92d:60057)

4. O. Kallenberg, Foundations of Modern Probability, 2nd ed., Springer-Verlag, New York, 2002. MR 1876169 (2002m:60002)

5. T. Konstantopoulos, A. Kyprianou, M. Sirviö, and P. Salminen, Analysis of stochastic fluid queues driven by local time processes, Adv. Appl. Probability 40 (2008), 1072-1103. MR 2488533 (2010d:60205)

6. T. Konstantopoulos and G. Last, On the dynamics and performance of stochastic fluid systems, J. Appl. Prob. 37 (2000), 652-667. MR1782443 (2001j:60168)

7. T. Konstantopoulos, M. Zazanis and G. de Veciana, Conservation laws and reflection mappings with an application to multiclass mean value analysis for stochastic fluid queues, Stoch. Proc. Appl. 65, No. 1 (1997), 139-146. MR1422884 (98k:60163)

8. T. Konstantopoulos, Open problems session of the workshop on "New Topics at the Interface between Probability and Communications", Isaac Newton Institute for Mathematical Sciences, Cambridge, UK, 14 January 2010.

9. D. Revuz and M. Yor, Continuous Martingales and Brownian Motion, Springer-Verlag, New York, 1999. MR 1725357 (2000h:60050)

10. A.V. Skorokhod, Stochastic equations for diffusions in a bounded region, Theory Probab. Appl. 6 (1961), 264-274. 
11. H. Tanaka, Stochastic differential equations with reflecting boundary condition in convex regions, Hiroshima Math. J. 9 (1979), 163-177. MR529332 (80k:60075)

12. W. Whitt, Stochastic-Process Limits, Springer-Verlag, New York, 2002. MR.1876437 (2003f:60005)

Department of Electrical Engineering and Computer Sciences, University of CaliFORNia, Berkeley, CALIFORNia 94720

E-mail address: ananth@eecs.berkeley.edu

Department of Mathematics, Uppsala University, Box 480, 75106 Uppsala, Sweden

E-mail address: Takis.Konstantopoulos@math.uu.se 